Conclusion/Implication Interdiction Project applies concepts of clinical epidemiology similar to in-patient hospital infection control processes and may help HIV/AIDS patients to achieve undetectable HIV viral load, reduce STD co-infection and HIV transmission. The findings suggest that epidemiologic work to reduce the spread of HIV, STD co-infection and resistant HIV must be an important component of HIV/AIDS programs and will require collaborative work by medical providers and intensive practical health education by knowledgeable HIV/AIDS health educator/epidemiologist.

\section{LBP-1.02 ACCEPTABILITY AND APPEAL OF INTERNET-BASED STI AND HIV TESTING AMONG A SAMPLE OF GAY MEN AND CLIENTS ATTENDING STI CLINICS IN BRITISH COLUMBIA}

doi:10.1136/sextrans-2011-050119.8

${ }^{1} \mathrm{~T}$ S Hottes, ${ }^{1} \mathrm{M}$ Gilbert, ${ }^{1} \mathrm{M}$ Bondyra, ${ }^{1} \mathrm{D}$ Haag, ${ }^{2} \mathrm{~J}$ Shoveller. ${ }^{1} B C$ Centre for Disease Control, Vancouver, Canada; ${ }^{2}$ University of British Columbia, Canada

Background Several jurisdictions globally have implemented internet-based STI and HIV testing with good uptake and reach into untested populations. In British Columbia, a similar program is under development to offer online access to chlamydia, gonorrhoea, syphilis, and HIV testing. This program aims to increase the frequency of testing in individuals at risk of infection and ease demand on clinic-based services by providing an accessible option for screening asymptomatic persons. We conducted a series of focus groups in spring 2011 to determine the acceptability of various aspects of the internet testing model and of the service overall. Methods Two types of prospective internet testing clients living in the Vancouver area were recruited for focus groups: self-identified gay/bisexual/two-spirit men (hereafter "gay men") and STI clinic clients. Participants were presented with a brief description of the internet testing model, after which a structured interview guide was used to address the following domains: willingness to provide personal information online, ways to engender trust in the service, comfort with different ways of delivering results, interest in specific features, appeal of the service, and willingness to use the service.

Results Most of the 31 participants to-date (ages $21-70$ years) were gay men (81\%) and reported post-secondary education (74\%). All were self-described active internet users and had been tested previously for HIV or other STI. Overall, the concept of internet testing was thought to be acceptable and most participants expressed interest in using the service when it becomes available. Participants indicated that internet testing would be convenient and could enhance the accessibility of STI testing, particularly for people living outside of urban centres. Some noted that an internet approach would also have the potential to allay embarrassment that can arise when seeking STI testing in a face-to-face clinical setting. There was strong interest in assuring anonymity for those using the proposed internet testing service. Participants also emphasised the importance of providing information related to HIV diagnosis and referrals to peer support and counselling for those waiting for and receiving test results.

Conclusions Internet testing was deemed to be acceptable and desirable and appears to hold promise as a means to complement to existing clinic-based STI testing services in Vancouver, Canada.

\section{LBP-1.03 "NO ONE WAS THERE TO CARE FOR US" ASHRAYA'S RESPONSE TO ITS MEMBERS' NEEDS IN TERMS OF CARE AND SUPPORT}

doi:10.1136/sextrans-2011-050119.9

C Chevrier. Université de Montréal, Montréal, Canada

Background Sex workers (SWs) have been identified as a "high-risk group" and considerable effort has been invested in promoting "safer sex" among this group. However, their specific concerns and identities vanish in broader public health policy discussions related to treatment and particularly care. Ashodaya Samithi, a SWs organisation based in Mysore, Karnataka, has been providing extensive care and support (C\&S) services to SWs and "non-SWs" living with HIV through their sub-wing organisation, Ashraya, since 2008. Considering the enduring stigma attached to SWs, what motivates individuals to seek health services from Ashraya?

Methods 40 in-depth interviews (including 10 with non-SWs) were conducted with Ashraya members in 2011 to explore how Ashraya responds to the needs of its members in terms of C\&S. Content analysis of the transcripts was completed to sort out themes.

Results Analysis shows that in spite of scarce resources, the accessibility, effective outreach efforts, and commitment and reliability of Ashraya's volunteers are major components of its growing popularity.

Conclusions In the near-absence of non-discriminatory, affordable C\&S for people living with HIV/AIDS, Ashraya offers much needed relief to its members. The potential role of SWs community-based organisations in providing $C \& S$, which goes beyond safer sex promotion, needs to be accounted for within national-level policy planning and in evaluating existing programs.

\section{LBP-1.04 INTERNATIONAL VALIDATION OF AMPLISENS NAAT SYSTEMS MANUFACTURED IN RUSSIA FOR DIAGNOSIS OF NEISSERIA GONORRHOEAE, CHLAMYDIA TRACHOMATIS, MYCOPLASMA GENITALIUM AND TRICHOMONAS VAGINALIS}

doi:10.1136/sextrans-2011-050119.10

${ }^{1} \mathrm{M}$ Domeika, ${ }^{2} \mathrm{~A}$ Savicheva, ${ }^{2} \mathrm{E}$ Shipitsyna, ${ }^{3} \mathrm{C}$ Y Chen, ${ }^{4} \mathrm{~J}$ Skov-Jenssen, ${ }^{5} \mathrm{O}$ Hjelmevoll, ${ }^{4} \mathrm{R}$ Ballard, ${ }^{6} \mathrm{M}$ Unemo. ${ }^{1}$ Uppsala University, Uppsala, Sweden; ${ }^{2}$ Institute of Obstetrics and Gynecology DI Ott, St Petersburg, Russian Federation; ${ }^{3}$ Centers for Disease Control and Prevention, Atlanta, Georgia, USA; ${ }^{4}$ State Serum Institute, Copenhagen, Denmark; ${ }^{5}$ University Hospital of North Norway, Tromso, Norway; ${ }^{6}$ Örebro University Hospital, Örebro, Sweden

Objectives Data regarding the performance characteristics of STI diagnostic tests used in Eastern Europe are very scarce. The objective of this study was to validate the AmpliSens test systems (Central Research Institute for Epidemiology, Moscow, Russia), compared to internationally acknowledged and validated tests, for genetic detection of $N$ gonorrhoeae (NG), C trachomatis (CT), $M$ genitalium (MG) and $T$ vaginalis (TV).

Methods In total, 319 females and 127 males were recruited. For evaluation of the TV tests, additional 33 patients with diagnosed trichomoniasis at dermatovenereological dispensaries (VD) were involved. Conventional AmpliSens PCR assay (cPCR), real-time PCR assay (rtPCR), and a real-time nucleic acid sequence-based amplification assay for detection of NG or CT were evaluated. Furthermore, AmpliSens cPCR and rtPCR for detection of MG or TV were tested. As international reference tests an internationally validated real-time $N$ gonorrhoeae porA pseudogene PCR, Cobas Amplicor CT PCR and LightMix 480HT CT PCR, MG rtPCR targeting the $\mathrm{MgPa}$ adhesin gene with positive results confirmed by two MG cPCRs targeting the $16 \mathrm{~S}$ rRNA gene and MgPa gene, and a TV rtPCR targeting a TV specific repeat DNA fragment, respectively, were used.

Results The overall prevalence of CT infection was $12.6 \%$. The AmpliSens CT NAATs and the reference methods displayed a high level of concordance $(97.9 \%-99.2 \%)$. The prevalence of NG was $2.7 \%$ and $16 \%$ among the urogenital and extragenital samples, respectively. The AmpliSens NG NAATs and the reference method 